\title{
ON A CLASS OF EXISTENCE THEOREMS IN DIFFERENTIAL GEOMETRY
}

\author{
BY T. Y. THOMAS AND JACK LEVINE
}

1. Introduction. Many problems in differential geometry require the integration of a mixed system of differential equations

$$
\begin{aligned}
& \frac{\partial u^{\alpha}}{\partial x^{i}}=\psi_{i}^{\alpha}(u, x), \\
& F_{0}(u, x)=0,
\end{aligned}
$$

which gives rise to an infinite sequence of sets of algebraic equations

$$
F_{1}=0, F_{2}=0, \cdots, F_{N}=0, \cdots,
$$

related to the system $\mathrm{E}$ by the following property.

$A$ necessary and sufficient condition for the existence of a solution of the system $\mathrm{E}$ is that there exist an integer $N$ such that the first $N$ sets of equations of the sequence (1) be algebraically consistent and that all their solutions satisfy the $(N+1)$ st set of equations of the sequence.

In many cases the functions $\psi$ and $F_{0}$ are linear and homogeneous in the unknowns $u$ with the result that the equations (1) are of a similar character; also the coefficients $A$ of the unknowns in (1) are the components of tensor differential invariants. In fact, the left members of each set of the equations (1) break up into the components of scalars and tensors. We shall confine our attention to systems $E$ of this type. Since such systems $\mathrm{E}$ always possess a trivial solution $u=0$, we shall mean by a solution $u(x)$ of $\mathrm{E}$ a non-trivial solution of this system.

A necessary and sufficient condition for the existence of a solution of the first $N$ sets of equations of the sequence (1) is the vanishing of their resultant system $R_{N}(A)$. For equations of the type under consideration in which the coefficients are real quantities, the solution whose existence is implied by the vanishing of the resultant system will be real.*

* When the equations (1) are linear and homogeneous in the unknowns $u^{\alpha}$, the resultant system $R_{N}$ can be taken to consist of the totality of all determinants of order $L$, equal to the number of unknowns $u^{\alpha}$, which can be formed from the matrix of the coefficients of the first $N$ sets of equations (1). 
By the above hypothesis concerning the tensor character of the equations (1), we can think of the $A$ 's as the coefficients of a set of multilinear forms $\Phi$ in one or more sets of cogredient and contragredient variables. Let $A \rightarrow \bar{A}$ as a result of a transformation of the variables of these forms. Then the vanishing of the resultants $R_{N}(A)$ implies the vanishing of the resultants $R_{N}(\bar{A})$ in the transformed coefficients $\bar{A}$, that is, the equations $R_{N}(A)=0$ are a set of invariant equations in the sense of the algebraic invariant theory. Now, by Gram's theorem,* the invariant equations $R_{N}(A)=0$ are equivalent to the identical vanishing of a set of covariants $\dagger$ of the forms $\Phi$. Hence the conditions on the components $A$ expressed by the equations $R_{N}(A)=0$ are equivalent to the vanishing of a set of tensor $\ddagger$ differential invariants $T(A)$ with components which are polynomials in the coefficients $A$.

2. The Resultant System. Let $L(\geqq 2)$ denote the number of unknowns $u$ in the system $\mathrm{E}$, and let the symbol $\left\{F_{N}\right\}$ represent the first $N$ sets of equations of the sequence (1). It will now be shown that the vanishing of the resultant system $R_{L}$ is necessary and sufficient for $\mathrm{E}$ to admit a solution.

Proof of the Necessity. If $E$ has a solution, the set $\left\{F_{L}\right\}$ is satisfied by this solution, so that the resultant system $R_{L_{\text {t }}}$ vanishes.

Proof of the Sufficiency. Suppose $R_{L}=0$. Then $\left\{F_{L}\right\}$ has a solution; in particular $\left\{F_{1}\right\}$ will have a solution. When the equations $F_{2}=0$ are added to the set $\left\{F_{1}\right\}$ two cases may arise:

$(\alpha)$ all solutions of $\left\{F_{1}\right\}$ satisfy $\left\{F_{2}\right\}$;

$(\beta)$ the set $\left\{F_{2}\right\}$ is consistent but all solutions of $\left\{F_{1}\right\}$ do not satisfy $\left\{F_{2}\right\}$.

If $(\alpha)$ occurs, then $\mathrm{E}$ admits a solution by the existence theorem stated in $\$ 1$. If $(\beta)$ occurs, we add the equations $F_{3}=0$ to the set $\left\{F_{2}\right\}$ and consider cases analogous to the above, that is, cases $(\alpha)$ and $(\beta)$ with $\left\{F_{1}\right\}$ replaced by $\left\{F_{2}\right\}$ and $\left\{F_{2}\right\}$ by $\left\{F_{3}\right\}$. Continuing in this manner, we must finally reach a set $\left\{F_{M}\right\}$ with

* See, for example, R. Weitzenböck, Invariantentheorie, 1923, p. 160.

$\dagger$ This is to be understood as including as a special case the vanishing of invariants of the forms $\Phi$.

$¥$ This is considered to include the scalar differential invariants. 
$M<L$ such that all solutions of $\left\{F_{M}\right\}$ satisfy $F_{M+1}=0$. In fact, either a set $\left\{F_{M}\right\}$ where $M<L-1$ is obtained such that all solutions of $\left\{F_{M}\right\}$ satisfy $F_{M+1}=0$, or else we arrive at the set $\left\{F_{L-1}\right\}$. But in this latter case there will be exactly $L-1$ independent equations in $\left\{F_{L-1}\right\}$, so that the equations $F_{L}=0$ cannot add new independent equations to the set $\left\{F_{L-1}\right\}$. Hence all solutions of $\left\{F_{L-1}\right\}$ will satisfy $F_{L}=0$, and the sufficiency is established.

3. Fields of Parallel Vectors. In the problem of determining the conditions under which a general affinely connected space admits a field of parallel contravariant vectors we arrive at the sequence

$$
\lambda^{j} B_{j k l}^{i}=0 ; \quad \lambda^{j} B_{j k l, m}^{i}=0 ; \quad \lambda^{j} B_{j k l, m, p}^{i}=0 ; \cdots
$$

corresponding to (1), where $B_{j k l}^{i}$ are the components of the curvature tensor $B$ and $B_{j k l, m}^{i}, B_{j k l, m, p}^{i}, \cdots$ are the components of the successive covariant derivatives of $B$. Here $L=n$. Hence we have the following theorem.

THEOREM. There exists a set of integral rational tensor differential invariants $P$ of a general affinely connected space such that the vanishing of the set $P$ is necessary and sufficient for the existence of a field of parallel contravariant vectors.

It is to be noted that the order in the derivatives of the components of the affine connection of any invariant in $P$ is $\leqq n$. Similar remarks apply to the existence of a field of parallel covariant vectors.

4. First Integrals of the Paths of an Affinely Connected Space. Consider an affinely connected space with symmetric affine connection $\Gamma$, and let us seek to determine a symmetric covariant tensor with components $a_{\alpha \beta} \ldots \gamma$ possessing the property that its covariant derivative vanishes, that is,

$$
a_{\alpha \beta \cdots \gamma, \delta}=0 .
$$

These equations correspond to the differential equations $E_{1}$; in this case the set $E_{2}$ does not appear. The number of unknowns $a_{\alpha \beta} \ldots{ }_{\gamma}$ in (2) is given by 


$$
L=\frac{(n+k-1) !}{(n-1) ! k !}
$$

where $k$ is the number of indices in the set $\alpha \beta \cdots \gamma$.

TheOREm. There exists a set of integral rational tensor differential invariants $I_{k}$ whose vanishing is necessary and sufficient for (2) to admit a solution.

The vanishing of the invariants $I_{k}$ is a sufficient condition for the existence of a first integral,

$$
a_{\alpha \beta} \cdots \gamma \frac{d x^{\alpha}}{d s} \cdot \frac{d x^{\beta}}{d s} \cdots \frac{d x^{\gamma}}{d s}=\text { const. }
$$

of the differential equations which define the paths of the affinely connected space. A necessary and sufficient condition for the existence of the first integral (3) is that

$$
P\left(a_{\alpha \beta} \cdots \gamma, \sigma\right)=0,
$$

where $P$ indicates the sum of the terms obtainable from the one in the parenthesis by a cyclic permutation of the indices $\alpha \beta \cdots \gamma \sigma$.

For linear first integrals we have the equations

$$
a_{\alpha, \beta}+a_{\beta, \alpha}=0 .
$$

Putting $a_{\alpha, \beta}=b_{\alpha \beta}$, we can deduce* as the system $\mathrm{E}_{1}$ :

$$
\begin{aligned}
\frac{\partial a_{\alpha}}{\partial x^{\beta}} & =a_{\sigma} \Gamma_{\alpha \beta}^{\sigma}+b_{\alpha \beta}, \\
b_{\alpha \beta, \gamma} & =a_{\sigma} B_{\gamma \alpha \beta}^{\sigma} .
\end{aligned}
$$

We obtain immediately from (5) the system $\mathrm{E}_{2}$ :

$$
b_{\alpha \beta}+b_{\beta \alpha}=0 .
$$

Corresponding to the equations (6) for the linear first integral, we have in the case of the quadratic first integral for the system $\mathrm{E}_{1}$ :

* O. Veblen and T. Y. Thomas, Transactions of this Society, vol. 25 (1923), p. 592; also L. P. Eisenhart, Non-Riemannian Geometry, p. 120. 


$$
\begin{aligned}
g_{i j, p} & =g_{i j p}, \\
g_{i j p, q} & =g_{i j p q}-g_{\alpha j} A_{i p q}^{\alpha}-g_{i \alpha} A_{j p q}^{\alpha}, \\
g_{i j p q, r} & =g_{\alpha \beta} G_{i j p q r}^{\alpha \beta}+g_{\alpha \beta \gamma} H_{i j p q r}^{\alpha \beta \gamma},
\end{aligned}
$$

where $G$ and $H$ are tensors; and for the system $\mathrm{E}_{2}$ :

$$
\begin{aligned}
& g_{i j}=g_{j i}, \quad g_{i j p}=g_{j i p}, \quad g_{i j p q}=g_{j i p q}=g_{i j p q}, \\
& g_{i j p q}+g_{j p i q}+g_{p i j q}=2\left(g_{i \alpha} A_{j p q}^{\alpha}+g_{j \alpha} A_{p i q}^{\alpha}+g_{p \alpha} A_{i j q}^{\alpha}\right), \\
& g_{i j p}+g_{j p i}+g_{p i j}=0 .
\end{aligned}
$$

A system $\mathrm{E}$ for first integrals (3) of the $k$ th degree can be obtained analogous to the above systems $\mathrm{E}$ for linear and quadratic integrals. Hence we have the following result.

THEOREM. There exists a set of integral rational tensor differential invariants $I_{k}^{*}$ of a space with symmetric affine connection such that the vanishing of the set $I_{*}^{*}$ is necessary and sufficient for the existence of a first integral of the kth degree.

The number of unknowns $L$ in the system (6) and (7) is equal to $n^{2}+n$. We can, however, eliminate the equations (7) from this system $\mathrm{E}$ by considering only the unknowns $b_{\alpha \beta}$ for which $\alpha>\beta$. For the equations (6), as so modified, the number of unknowns $L$ is equal to $\left(n^{2}+n\right) / 2$. Hence the maximum order of the differential invariants of a set $T(A)$ as predicted by the integer $L$ according to the discussion of $\$ 2$ need not necessarily be attained.

5. Groups of Motions. A metric space $V_{n}$ will admit a group of motions with contravariant components $\xi^{\alpha}$ if, and only if, the equations of Killing are satisfied, that is,

$$
\xi_{\alpha, \beta}+\xi_{\beta, \alpha}=0
$$

where $\xi_{\alpha}$ is the associated covariant form of the components $\xi^{\alpha}$. Since the equations (10) are of the same form as (5), it follows that the vanishing of the above set of differential invariants $I_{1}^{*}$ for the space $V_{n}$ is a necessary and sufficient condition for this space to admit a group of motions.

Now consider the sequence (1) constructed from the equations (6) and (7) with reference to the space $V_{n}$. If there exists an integer $M$ such that the ranks of the matrices of the sets $\left\{F_{M}\right\}$ and $\left\{F_{M+1}\right\}$ are $L-r$, where $L=n^{2}+n$ denotes the number 
of unknowns, then the $V_{n}$ admits a group $G_{r}$ of motions. Let $\left\{D_{r}\right\}$ represent the equations obtained by equating to zero all determinants of order $L-r+1$ in the matrix of the set $\left\{F_{L}\right\}$. The equations $\left\{D_{r}\right\}$ imply the vanishing of the resultant system $R_{L}$ and hence by the argument of $\$ 2$ there exists an integer $M$ such that all solutions of $\left\{F_{M}\right\}$ satisfy $\left\{F_{M+1}\right\}$. But there must exist at least $r$ independent solutions of $\left\{F_{M}\right\}$ in consequence of the conditions $\left\{D_{r}\right\}$. Hence the conditions $\left\{D_{r}\right\}$ are sufficient for the existence of a group of motions in $r$ or more parameters. Evidently the conditions $\left\{D_{r}\right\}$ are necessary for the existence of such a group of motions. Moreover, the equations $\left\{D_{r}\right\}$ form an invariant system; this follows from the fact that $\left\{D_{r}\right\}$ assures the existence of $s \geqq r$ independent solutions of $\left\{F_{L}\right\}$ and hence of the transformed equations $\left\{\bar{F}_{L}\right\}$, which implies the existence of the corresponding equations $\left\{\bar{D}_{r}\right\}$. Hence there exists a set of integral rational tensor differential invariants $H_{r}$ whose vanishing is equivalent to the conditions $\left\{D_{r}\right\}$.

A group of motions of a $V_{n}$ involves at most $J=\left(n^{2}+n\right) / 2$ parameters; also when $V_{n}$ admits a $G_{J}$ of motions the space is of constant curvature.* Hence the above considerations show the existence of $J$ sets of differential invariants $H$, namely

$$
H_{1}, H_{2}, \cdots, H_{J}
$$

such that the vanishing of any set of these invariants implies the vanishing of all preceding sets. We can now state the following theorem.

THEOREM. The vanishing of the set of differential invariants $H_{r}$ is a necessary and sufficient condition for a metric space $V_{n}$ to admit a group of motions in $R$ parameters, where $r \leqq R \leqq J$. Also a necessary and sufficient condition for the existence of a $G_{r}$ of motions is the vanishing of the set $H_{r}$ and non-vanishing of the set $H_{r+1}$.

It is known that there exists no $G_{J-1}$ of motions of a $V_{n} . \dagger$ This fact taken in connection with the above theorem shows that the vanishing of the set $H_{J-1}$ implies the vanishing of the set $H_{J}$. Moreover, the conditions imposed by equating the invariants $H_{\boldsymbol{J}}$ to zero must be equivalent to the conditions

* See, for example, L. P. Eisenhart, Riemannian Geometry, 1926, p. 239.

† L. P. Eisenhart, loc. cit., p. 246. 


$$
B_{\alpha \beta \gamma \delta}=K\left(g_{\alpha \gamma} g_{\beta \delta}-g_{\alpha \delta} g_{\beta \gamma}\right),
$$

which characterize a space $V_{n}$ of constant curvature $K$.

Theorems analogous to the above can be stated concerning the existence of affine and projective collineations in an affinely connected space.

6. Metric Representation of an Affinely Connected Space. It was shown in $\$ 4$ that a necessary and sufficient condition for the system

$$
\frac{\partial g_{\alpha \beta}}{\partial x^{\gamma}}-g_{\sigma \beta} \Gamma_{\alpha \gamma}^{\sigma}-g_{\alpha \sigma} \Gamma_{\beta \gamma}^{\sigma}=0
$$

to admit a solution in the symmetric unknowns $g_{\alpha \beta}$ is the vanishing of the set of invariants $I_{2}$. If the system (12) admits a solution $g_{\alpha \beta}(x)$ such that the determinant $\left|g_{\alpha \beta}\right|$ does not vanish identically, the affinely connected space $S$ is said to reduce to a metric space based on the quadratic differential form

$$
\phi=g_{\alpha \beta} d x^{\alpha} d x^{\beta},
$$

or in other words to admit an $n$-dimensional metric representation.

Now suppose that (12) possesses a solution $g_{\alpha \beta}$ such that the rank of the matrix $\left\|g_{\alpha \beta}\right\|$ is $r$, where $1 \leqq r<n$. Without loss of generality it can then be assumed that $\left\|g_{\alpha \beta}\right\|$ contains a nonvanishing determinant of order $r$ in its upper left hand corner. Then

$$
g_{\alpha b}=g_{a b} A_{\alpha}^{a}, \quad g_{\alpha \beta}=g_{a \beta} A_{\alpha}^{a},
$$

$(a, b=1, \cdots, r ; \alpha, \beta=r+1, \cdots, n)$. It has been shown that (13) can be reduced to the form

$$
\sum_{a, b=1}^{r} h_{a b}(y) d y^{a} d y^{b}
$$

by a coordinate transformation $x \rightarrow y$ if, and only if, the following equations are satisfied :*

* T. Y. Thomas, Proceedings of the National Academy of Sciences, vol. 20 (1934), pp. 215-219. 


$$
\frac{\partial A_{\alpha}^{a}}{\partial x^{b}} \cdot A_{\beta}^{b}-\frac{\partial A_{\beta}^{a}}{\partial x^{b}} \cdot A_{\alpha}^{b}-\frac{\partial A_{\alpha}^{a}}{\partial x^{\beta}}+\frac{\partial A_{\beta}^{a}}{\partial x^{\alpha}}=0,
$$

$(a, b=1, \cdots, r ; \alpha, \beta=r+1, \cdots, n)$. On substituting for the $A$ 's their values in terms of the $g_{\alpha \beta}$ as obtained from (14) and making use of (12), we find that the equations (16) are satisfied identically. Hence the transformation of (13) to the form (15) is possible.

Under the above hypothesis that the rank of $\left\|g_{\alpha \beta}\right\|$ is $r<n$, it is easily seen from (12) that

$$
C_{\alpha k}^{a}(y)=0
$$

$(a=1, \cdots, r ; \alpha=r+1, \cdots, n ; k=1, \cdots, n)$, where the $C$ 's are the components of the connection in the $y$ coordinate system. It likewise follows from (12) and (17) that

$$
\frac{\partial h_{a b}}{\partial y^{\alpha}}=0
$$

$(a, b=1, \cdots, r ; \alpha=r+1, \cdots, n)$, that is, the coefficients $h_{a b}$ in (15) depend on the variables $y^{1}, \cdots, y^{r}$ alone.

The correspondence

$$
y^{1}, \cdots, y^{r}, y^{r+1}, \cdots, y^{n} \rightarrow y^{1}, \cdots, y^{r}
$$

defines an isomorphism between the space $S$ and an $r$-dimensional space $S^{*}$. Let the metric of $S$ be defined by the form (13) and the metric of $S^{*}$ by the form (15). Then the distance between any two points $P$ and $Q$ in $S$ measured along a curve $C$ joining these points is equal to the distance between the corresponding points $P^{*}$ and $Q^{*}$ measured along the corresponding curve $C^{*}$ in $S^{*}$. The space $S^{*}$ will therefore be said to be an $r$-dimensional metric representation of the affine space $S$.

TheOREM. A necessary and sufficient condition for the space $S$ to admit an $r$-dimensional metric representation $S^{*}$, where $1 \leqq r \leqq n$, is the vanishing of the set of differential invariants $I_{2}$.

Princeton University 